\title{
SOROTERAPIA INTRATECAL NO TÉTANO. REVISÃO
}

\author{
Anna Sara Shaterman LGVIN, Antonio Alci BARONE \& Mario SHIROMA
}

\section{R ES U MO}

Devido à importância do tétano nos países em desenvolvimento, foi feita uma revisão da literatura recente abordando aspectos históricos e bases fisiopatológicas do uso da soroterapia intratecal no tratamento do tétano. Discutese principalmente a eficácia dessa terapêutica envolvendo o uso da antitoxina heteróloga e homóloga, associada ou não à antitoxina e a corticosteróides por via sistêmica. São abordadas também as complicaçōes do uso da antitoxina por via intrarraquideana que são, em geral, leves e transitórias. As tentativas de introduzir definitivamente a administração intratecal da antitoxina tetânica, na terapêutica do tétano, são precoces, uma vez que estudos existentes até o momento tem resultados contraditórios e muitas variáveis envolvidas.

\section{UNITERMOS: Tétano - terapêutica; Antitoxina tetânica - Administração in-} tratecal; Uso terapêutico - Administração e dosagem.

\section{N T R O D U G A O}

A utilização de soro anti-tetânico, por via intrarraquidiana no tratamento do tétano, é um procedimento antigo. Desde o final do século passado e o início deste, há relatos do uso deste procedimento.

PENNA 27, em 1902, descreve o uso de "anti-soro" subaracnoideo em cinco pacientes com tétano grave, sendo que três deles sobrevive. ram e dois faleceram em decorrência de pneumonia, após a melhora do tétano.

ROGERS 31 , em 1905, descreve o tratamento com "anti-soro" (termo utilizado pelo próprio autor) por via intrarraquidiana lombar, assa ciado à via intraneural, endovenosa e subcutânea, com boa resposta em quatro de sete pacientes.

A justificativa teorica para o uso da via Intratecal seria a neuralização da toxina livre no líquido céfalo-raquidiano $8,29,48$ antes que esta pudesse se fixar no sistema nervoso cen. tral (SNC). A baixa penetração da antitoxina pela barreira hemato-liquorica, quando introduzida por via intramuscular ou endovenosa, é demonstrada por ILDIRIM 18 e por ARNAUD et al 1 , usando antitoxina marcada com iodo radioativo, embora eles tenham pequeno número de pacientes em suas experiências.

PATEL ${ }^{26}$, em 1984, colhendo amostras de liquor e sangue de pacientes tetânicos à admissão hospitalar e antes de qualquer terapêutica especifica, demonstra que há baixos níveis de toxina nesses fluidos. Observa ele, que em apenas quatro dos 105 casos estudg. dos há presença de toxina livre no liquor. Fm razão dessa observação, questiona-se: a antitoxina, uma vez ultrapassada a barreira hemato-liquórica, poderia neutralizar a toxina já fixada no Sistema Nervoso?

ARNAUD et al. 1 , em seu trabalho, aven. tam a possibilidade da antitoxina, por via in

Trabalho realizado no Departamento de Doenças Infecciosas e Parasitárias, da Faculdade de Medicina da Universidade de São Paulo, Brasil.

Endereço para correspondêncla: Dra. Anna Sara Shafferman Levin - Rua Harmonia, 564 - apto. 52 - 05435 Säo Paulo, sP - Brasil. 
LEVIN, A. S. S.; BARONE, A. A. \& SHIROMA, M. - Soroterapia intratecal no tétano. Revisăo. Rev. Inst. Med. trop.

São Paulo, 29:25ุ5-267, 1987.

tratecal, se fixar no parênquima nervoso. Em 1978, HANAUSKE et al. ${ }^{15}$, usando antitoxina tetânica fracionada, marcađa, $\mathbf{F}\left(a b^{\prime}\right){ }^{2-125}$, injetada por via intratecal em gatos, notam que a sua fixação ocorre, em ordem decrescente: na duramater, gânglios nervosos dorsais e nerivo ciático, sendo que não há fixação em raízes nervosas ventrais, dorsais ou segmentos do cordão espinal. WELLHONER et al ${ }^{49}$, em 1984, demonstram que a imunoglobulina antitetâni. ca livre não consegue penetrar a membrana plasmática neuronal, em ratos, e, portanto não tem ação sobre a toxina que se encontra no motoneurônio.

Além disso, em Mesa Redonda realizada durante a Quarta Conferência Internacional sobre Tétano ${ }^{8}$, em 1975 , é levantada a possi. bilidade teórica de haver sítios diferentes na toxina para a ligação com a antitoxina e para o. SNC, e que a antitoxina, mesmo ligada à toxina, não seria efetiva para desligála de seu receptor nervoso, ou para diminuir sua açäo. E sugerida, também, a existência de antitoxinas diferentes para diversos receptores na toxina, o que explicaria, talvez a variabilidade encontrada entre os diversos estudos de autores diferentes.

Existem, também, estudos em animais para testar a eficácia dessa via em tétano experimental.

Já em 1914, PARK \& NICOLL 25 fazem experiências com tétano prơvocado em cobaias, em que demonstram a superioridade do uso đa antitoxina por via espinal em relação à via intracardíaca, subcutânea ou intraneural. Em 1917, SHERRINGTON ${ }^{37}$, em macacos, também demonstra a vantagem da via intratecal, lombar, sobre a via subcutânea, intramuscular ou endovenosa da antitoxina, desde que administrada precocemente na doença.

SMITH ${ }^{40}$, em 1966, demonstra vantagem da via intracerebral sobre a intraperitoneal na administração da antitoxina, em tétano expe. rimental de ratos brancos. Essa vantagem 6 devida à presença da barreira hemato-liquórica, uma vez que a concentração de antitoxina intracerebral, com uma injeção intraperitoneal de 3.000 UI é, somente, comparável a 30 UI por via intracerebral, após seis horas da injeção.

Ha, entretanto, trabalhos com resultados conflitantes como o de FLOREX \& FILDES?, em 1927, que usam coelhos e não conseguem obter diferença significativa de resultados entre o uso da antitoxina intratecal e a endovenosa.

Após o entusiasmo no início do século, o uso da antitoxina por via intratecal $\varepsilon$ abandonada devido ao grande número de complicações e sua gravidade, conforme refere SPAETH ${ }^{41}$, em 1941, que se coloca contra essa prática. Essas complicaçōes talvez possam ser atribuidas à insuficiente purificação đa antitoxina usada.

Somente ao final da década de 60 e, mais ainda, a partir de 1970, é retomado o estudo nesse sentido.

Emm razão das inúmeras controvérsias exis. tentes e dada a importância do tétano nos países em desenvolvimento, inclusive o nosso, fazse aqui um levantamento daquilo que se en. contra, na literatura mais recente, sobre o uso clínico da soroterapia intratecal no tratamento do tétano.

\section{REVISAO DA LITERATURA REFERENTE AOS ESTUdos CLINICOS MAIS RECENTES}

Encontram-se, na literatura mais recente; estudos usando vários tipos de antitoxina te tânica por via intratecal, seja heteróloga, geralmente de origem equina (SAT), como homóloga, imunoglobulina humana específica (TIG). Em alguns estudos clínicos, utiliza-se antitoxina, tanto homóloga quanto heteróloga, fracionáada, ou seja, fraçóes $F(a b)^{2}$ e $F\left(a b^{\prime}\right)$, considerando a possibilidade teórica le facilidade maior na penetração dessas frações, de menor peso molecular, no SNC.

Alguns autores preconizam o uso de corticóide sistêmico (betametasona) no tratamento do tétano 34,36 , assim como administração intratecal (prednisolona, dexametasona, hidrocortisona) para diminuir os efeitos colaterais, como a irritação meníngea, provocados pela injeção da antitoxina, principalmente a heteróloga e a de preparados contendo preservati. vos 20 .

Encontram-se estudos que usam a antitoxina por via raquidiana isolada, ou associada a corticóides sistêmicos ou intratecais.

\section{A - $O$ uso da antîtoxina homóloga:}


LEVIN, A. S. S.; BARONE, A. A. \& SHIROMA, M. - Soroterapia intratecal no tétano. Revisäo. Rev. Inst. Med. trop. São Paulo, 29:255-267, 1987.

Os estudos que comparam o uso da Imu. noglobulina humana específica (TIG) somente por via intratecal, em relação ao seu uso sis- têmico, intramuscular, estão resumidos na Ta. bela I.

T A B E I A I

Resultados de estudos realizados com imunoglobulina humana especifica (TIG) por via intratecal isolada, compilados ia Iiteratura

\begin{tabular}{|c|c|c|c|c|}
\hline & Autor (Local) & BOLOT (França) a, 4 & DIOP-MAR (Senegal) 4,6 & GUPTA (fndia) ${ }^{14}$ \\
\hline & Ano & 1975 & 1975 & 1980 \\
\hline & N.o de casos & 45 & 147 & 97 \\
\hline & Idade & Idosis & Jovens ( 35 neonatos) & $\begin{array}{l}\text { a partir de } 12 \text { arios } \\
\text { (média }=15 \text { anos) }\end{array}$ \\
\hline & $\begin{array}{l}\text { Dose TIG } \\
\text { Intratecal }\end{array}$ & $1.000 \mathrm{UI}$ & 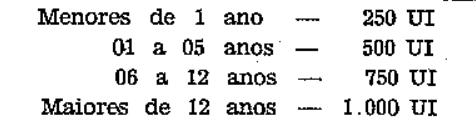 & $250 \mathrm{UI}$ \\
\hline & Via & Cisternal óu Lombar & Geralmente Cisternal & ? \\
\hline & Mortalidade & $33,3 \%$ & $33 \%$ & $2 \%$ \\
\hline $\begin{array}{l}\text { 田 } \\
9\end{array}$ & $\begin{array}{l}\text { Controle mortalidade } \\
\text { (N.० total casos) }\end{array}$ & $40,5 \%$ & $49 \% \quad(118)$ & $21 \% \quad(48)$ \\
\hline $\begin{array}{l}\text { P } \\
\text { - } \\
z \\
0 \\
0 \\
0\end{array}$ & $\begin{array}{l}\text { Dose TIG } \\
\text { Sistêmica e via }\end{array}$ & 1.500 UI IM & 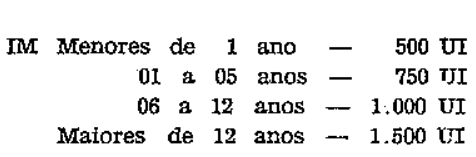 & 1.000 UI IM \\
\hline
\end{tabular}

$\mathrm{IM}=$ Intramuscular

BOLOT et al. 3,4, na França, em centros com recursos terapêuticos avançados, Terapia Intensiva, e com pacientes, na sua maioria idosos, nāo conseguem encontrar diferença significativa entre os resultados obtidos pelas duas vias.

O mesmo estudo realizado simultaneamente em Dakar 4,5, com recursos hospitalares e de pessoal paramédico limitađos, obtém resultados melhores pela via intratecal em relação à sistêmica, principalmente em pacientes menores de cinco anos ou com mais de 20 anos de idade.

GUPTA et al. ${ }^{14}$, na fnđia, tem resultados muito melhores pela via intratecal, com $2 \%$ de mortalidade em tétano do adulto, porém somente são incluidos nesse estudo, pacientes que não tem espasmos à internaçăo, isto é, tétano leve ou tétano inicial. Os autores acre. ditam no benefício dessa via somente em formas leves ou iniciais da doença. Nesse grupo de estudo $7 \%$ evoluem com espasmos, enquanto no grupo controle $31 \%$ apresentam espasmos.
A Tabela II resume os estudos em que se associam TIG por via intratecal e antitoxina, tanto TIG quanto SAT, por via sistêmica.

LIST 22 , na Austria, obtém bons resultados em dez pacientes, na maioria idosos, com a associação de TIG intratecal à terapêutica anteriormente por ele usada, dispondo de recursos de Terapia Intensiva. O número de pacientes, no entanto, é ainda pequeno para conclusōes.

SEDAGHATIAN ${ }^{35}$, no Irã, e VAKrI et al. 44,45,46, na Inđđia, não encontram vantagens da associação da antitoxina por via intrarraquidiana à via sistêmica tendo o primeiro estudado tétano neonatal, e o segundo, tétano em maiores de três anos de idade.

Há ainđa alguns relatos de casos que apontam favoravelmente ao uso da via intrate. call 16,17.

Em 1980, VERONESI et al. ${ }^{47}$ realizam um estudo com o uso de TIG fracionada, $F(a b)^{2}$, e também não chegam a conclusão definitiva 
LEVIN, A. S. S.; BARONE, A. A. \& SHIROMA, M. - Soroterapia intratecal no tétano. Revisăo. Rev. Inst. Med. trop. Săo Paulo, 29:255-267, 1987.

T A B E L A II

Resultados de estudos realizados com imunoglobina humana específica por via intratecal (TIG) associada à vía sístêrnica, compilados da literatura

\begin{tabular}{|c|c|c|c|}
\hline Autor (Local) & LIST (Âusțria) & SEDAGHATIAN (Iran) ${ }^{25}$ & VAKH (fndia) 44, 45,46 \\
\hline Ano & 1978 & 1979 & 1979 \\
\hline N.O de casos & 10 & 30 & 60 \\
\hline Idade & Adultos (Média 61 anos) & neonetos & Maiores de 3 anos \\
\hline $\begin{array}{l}\text { Dose TIG } \\
\text { Intratecal (Via) }\end{array}$ & $200 \mathrm{UI}$ & $150 \mathrm{UI}$ & 250 UI (cisternal) \\
\hline $\begin{array}{l}\text { SAT } \\
\text { Sistêmico Dose e Via }\end{array}$ & - & $\begin{array}{lll}20.000 & \text { UI } & \text { IM } \\
20.000 & \text { UI } & \mathrm{EV}\end{array}$ & 10.000 UI EV \\
\hline $\begin{array}{l}\text { TIG } \\
\text { Sistêmico }\end{array}$ & $60.000 \mathrm{UI}$ & - & 一 \\
\hline $\begin{array}{l}\text { Corticóide } \\
\text { Sistêmico }\end{array}$ & - & - & - \\
\hline $\begin{array}{l}\text { Corticóide } \\
\text { Intratecal }\end{array}$ & 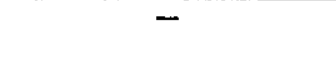 & - & - \\
\hline Mortalidade & $0 \%$ & $43 \%$ & $33 \%$ \\
\hline $\begin{array}{ll}\text { ra } & \text { Controle } \\
\text { A } & \text { Mortalidade } \\
0 & \text { (N. total casos) }\end{array}$ & Menor de $20 \%$ (46) & $50 \% \quad(30)$ & $23 \%(60)$ \\
\hline $\begin{array}{l}\text { SAT Sistêmico } \\
\text { 乙 Dose e Via }\end{array}$ & - & $\begin{array}{l}20.000 \text { UI IM e } \\
20.000 \text { UI EV }\end{array}$ & $10.000 \mathrm{UI} \mathrm{EV}$ \\
\hline $\begin{array}{l}\text { TIG Sistêmico } \\
\text { Dose e Via }\end{array}$ & $60.000 \mathrm{UI}$ & - & 一 \\
\hline
\end{tabular}

SAT = Antitoxina heteróloga de origem equina

IM $=$ Intramuscular

\section{B - O uso da antitoxina heteróloga:}

A Tabela III resume os resultados dos estudos que comparam o uso isolado de antitoxina tetânica de origem equiina (SAT) por via intrarraquidiana com o seu uso sistêmico.

As doses intratecais usadas são muito variáveis.

GALLAIS et al., da Costa do Marfim, em dois estudos 10,11 obtém mortalidade de $21 \%$ e $28 \%$, comparada a dados prévios de morta. lidade do mesmo serviço, que era de $40 \%$ a $50 \%$. O seu trabalho mais recente, em $1984{ }^{12}$, com uma casuística bem maior, demonstra resultados semelhantes aos anteriores. Nesses estudos usa-se corticóide somente por via in. tratecal.

DIOP-MAR et al. 6,7, sem o uso da associa̧̧ão com corticóides, obtém mortalidades de $23,14 \%$ e $31,08 \%$, sendo que, no segundo estudo, a mortalidade obtida no grupo controle $\delta$ de $42,46 \%$.
A Tabela IV resume os estudos clínicos em que se usa a via intratecal associada à via sistêmica do SAT, com ou sem corticóides. Verifica-se, nessa tabela, que os resultados são conflitantes.

Entre os resultados de estudos realizados com tétano neonatal, a mortalidade varia de $10,7 \%$ a $63,4 \%$.

SINGF et al. 38, na fndia, estudando tótano neonatal, encontram resultados significativamente melhores utilizando a via intratecal associađa à terapêutica sistêmica, porém somente quando a administração é iniciada até 24 horas após o aparecimento de espasmos.

KESWANI et al. 21, na fndia, em tétano do adulto, consideram que essa associaçăo das vias intratecal e sistêmica do SAT, fornece bons resultados quando usada antes de 48 ho. ras do inicio dos espasmos. 


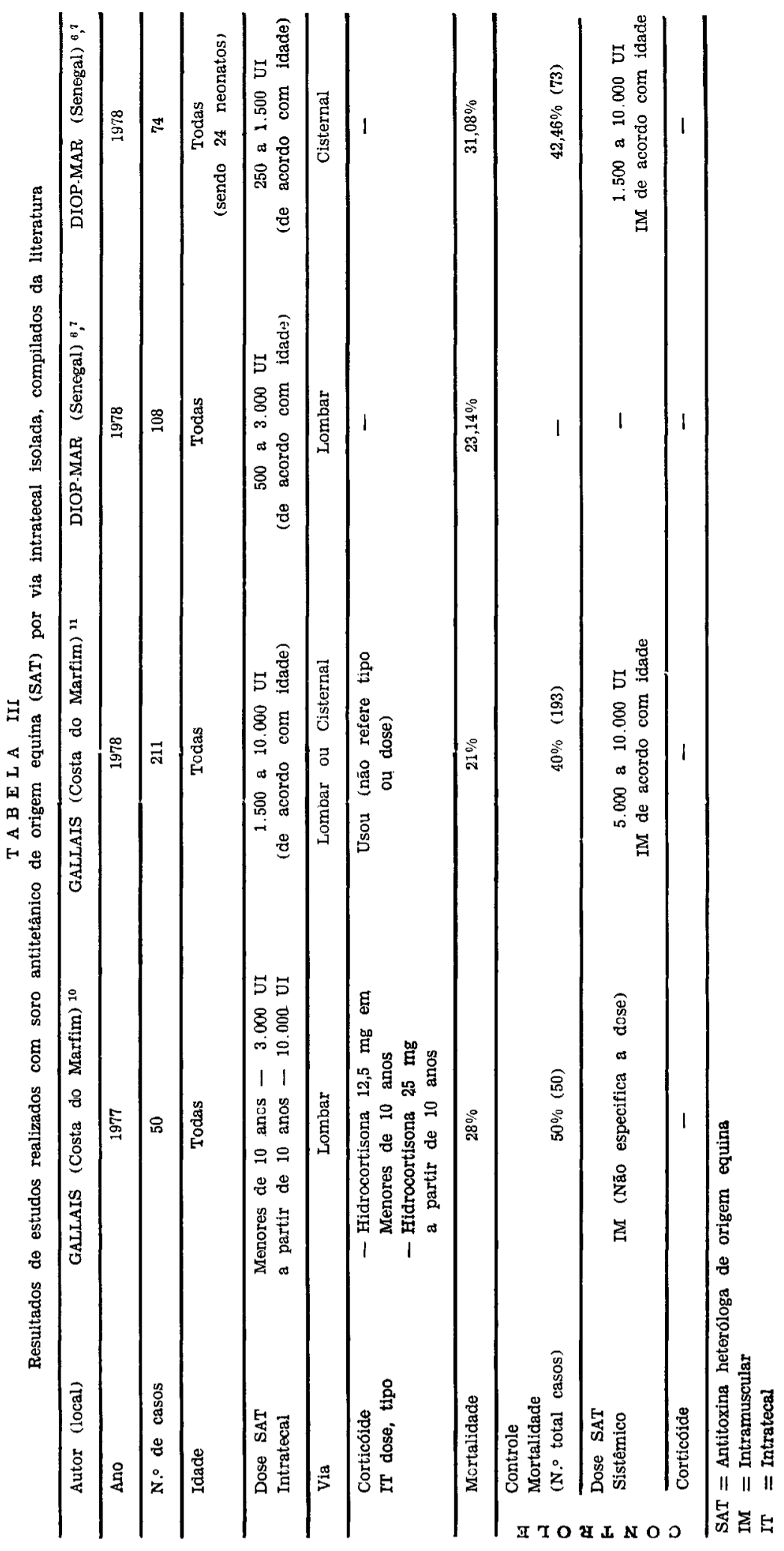



LEVIN, A. S. S.; BARONE, A. A. \& SHrROMA, M. - Soroterapia intratecal no tétano. Revisão. Rev. Inst. Međ. trop.
Șão Paulo, 29:255-267, 1987.

T A B E I A IV (1.a parte)

Resultados de estudos realizados com soro antitetânico de origem equina (SAT) por via intratecal associado à via sistêmica, compilados da literatura

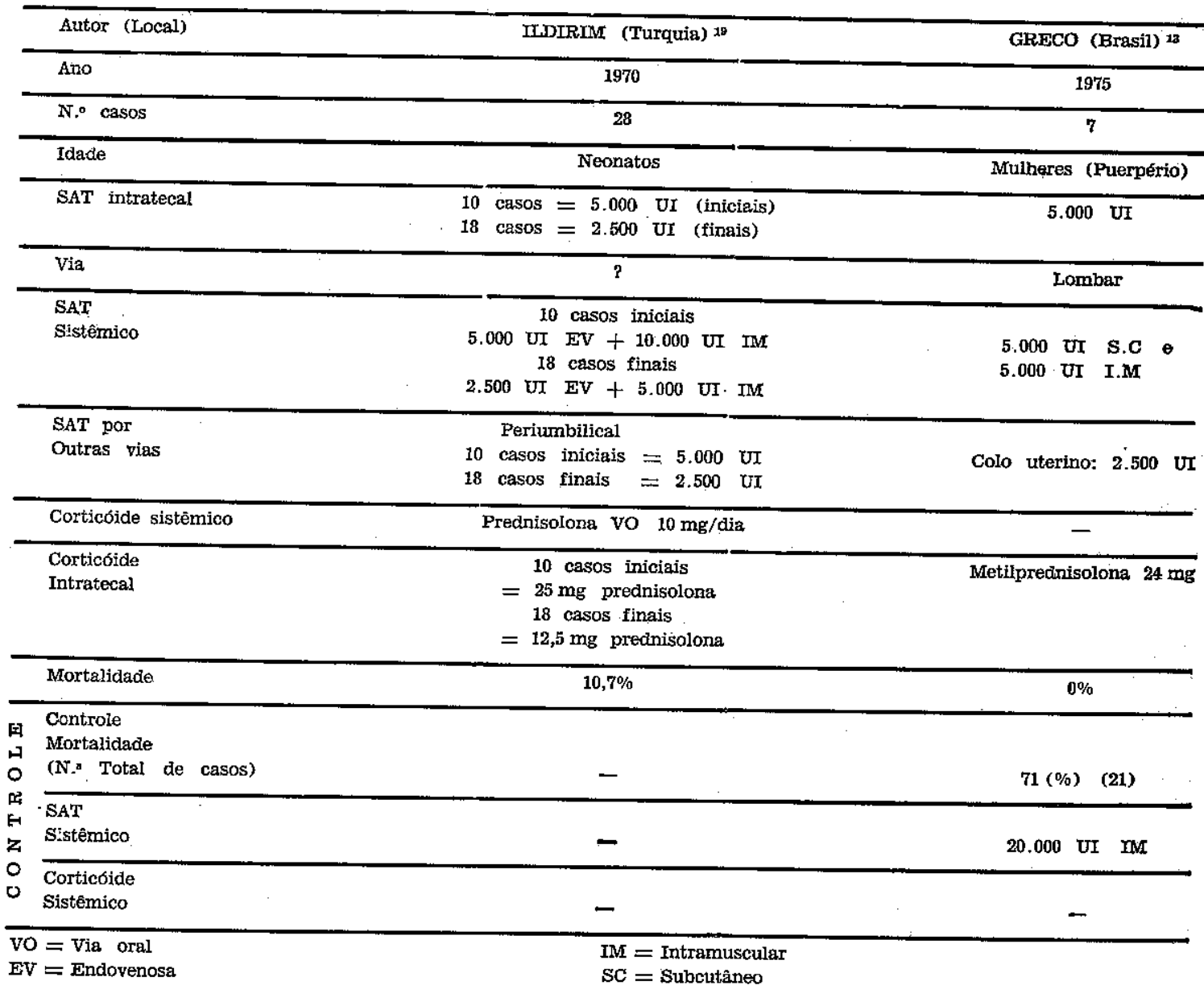

Parte dos estươs consultados não aponta uma vantagem significativa do uso da via intratecal 2,23,43.

ILDIRIM ${ }^{18}$, GRECO ${ }^{13}$ e ONUORA et al. ${ }^{24}$ não tem propriamente grupos controle, uma vez que o primeiro compara os seus resultados atuais com os de dez casos prévios do mesmo serviço e que não săo tratados de ma. neira semelhante. GRECO ${ }^{13}$, do Brasil, usa um grupo controle de tétano puerperal em que não é feita a limpezas do foco, curetagem uterina, adequadamente; em mais da metade dos casos. ONUORA et al, ${ }^{24}$, tem grupo controle retrospectivo de 30 casos de tétano do adulto, mas não.há dados quanto ao tratamen. to utilizado nesse grupo.
SANDERS et 81.32 , em 1977, estudando té tano do adulto, na fndia, encontram, mortalidade de $5 \%$ e $8 \%$ nos grupos de estudo contra $16 \%$ e $14 \%$ nos grupos controle. Essa. điferença não é significativa considerando os estudos em separado, porém quando se com. para o número total de pacientes que receberam SAT por via intratecal, com o total daqueles dos grupos controle, passa a haver uma diferença significativa.

VERONESI et al. ${ }^{48}$, em 1983, utiliza em tétano do adulto, SAT de origem equina, fracionado, $F\left(a b^{\prime}\right)$, admitindo bom resultado, sugerindo estudos complementares para se chegar a conclusões mais concretas.

O único estudo que compara SAT e TIG, realizado por ILDIRIM ${ }^{20}$, não encontra dife. 
LEVIN, A. S. S.; BARONE, A. A. \& SHIROMA, M. - Soroterapia intratecal no tétano. Revisão. Rev. Inst. Med. trop. São Paulo, 29:255-267, 1987.

TA B E LiA IV (2." parte)

Resultados de estudos realizados com scro antitetânico de origem equina (SAT) por via intratecal associada à via sistêmica, compilados da literatura

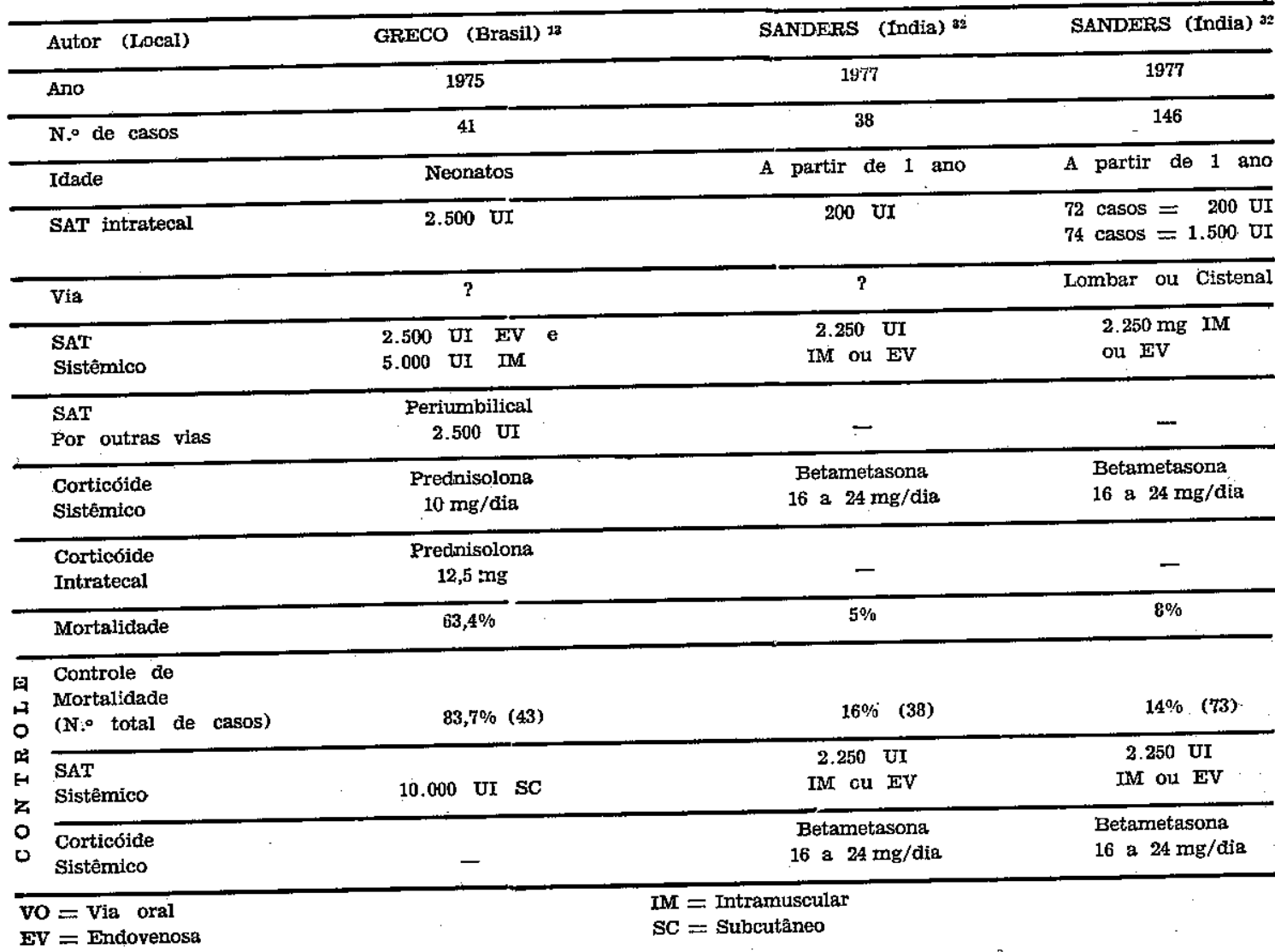

renças significativas entre os resultados dos dois grupos.

\section{G - $O$ uso de corticóide por via sistêmica:}

O estudo de SANDERS et al. ${ }^{32}$, em 1977, com tétano do adulto, obtém mortalidade nos grupos de estudo de $5 \%$ e $8 \%$, contra $16 \%$ e $14 \%$, nos grupos controle. Essa mortalidade é bastante baixa principalmente considerando nẩo haver recursos de Terapia Intensiva ou cuidados de enfermagem contínuos. Esses autores usam betametasona por via sistêmica, à qual atribuem seus bons resultados 33,34.

THOMAS et al. ${ }^{43}$, na fndia, em adultos, encontram vantagem no uso de betametasona por via sistêmica associada à antitoxina por via sistêmica e por via intratecal, sobre o uso sistêmico isolado da antitoxina. Nâo é possivel tirar conclusôes, nesse trabalho, quando se compara a associação ou não da betametasona à administração conjunta do SAT por via intratecal e sistêmica.

Em 1983, NEEQUAYE et al. 23, em Ghana, em neonatos, fazem estudo comparativo com e sem o uso de betametasona por via sistêmica e não encontram resultados melhores com o uso do corticóide.

\section{D - Posologia de antitoxina por via in- tratecal:}

Com a antitoxina homóloga, há dois estudos: VAKIL et al. ${ }^{45}$ que, em tétano do adulto, nắo mostram diferença de resultados entre 0 uso de 250 UI e 1.000 UI; e BOLOT et al. 4 que não encontram diferenças importantes entre o uso de $1.000 \mathrm{UI}$ e $\mathbf{2 . 0 0 0} \mathrm{UI}$, em pacientes em Dakar. Demonstram, porém, resultados melhores com a dose maior usada em centros franceses. Esses autores advogam o uso de 

LEVIN, A. S. S.; BARONE, A. A. \& SHIROMA; M. - Soroterapia intratecal no tétano. Revisăo. Rev. Inst. Med. trop.
Säo Paulo, 29:255-267, 1987.

T A B E I. A IV (3.* parte)

Resultados de estudos realizados com soro antitetânico de origem equina (SAT) por via intratecal associađa à via sistêmica, compilados da literatura

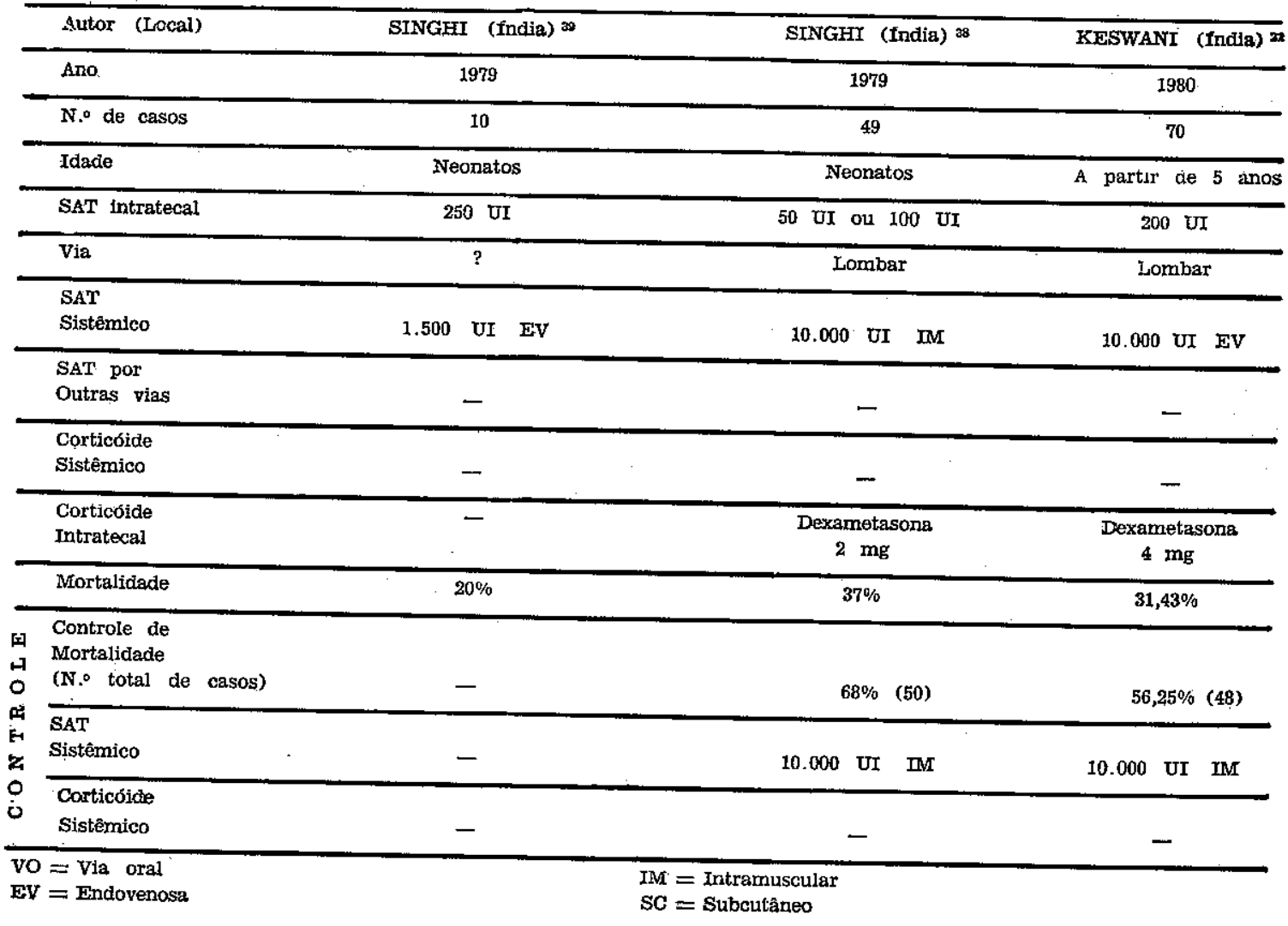

dose por quilograma de peso, mas não propõem uma posologia.

Com SAT, de origem equiina, há dois estudos; SANDERS et al. ${ }^{32}$, em tétano do adulto, comparam 200 UI e 1.500 UI; e SINGH et al. ${ }^{38}$, em neonatos, comparam 50 UI e 100 UI. Em ambos os estuđos a mortalidade não varia com a dose utilizada; no entanto, SANDERS et al. 32, demonstram que o tempo até o obito no grupo que utiliza 200 UI é o dobro daquele que usa $1.500 \mathrm{VI}$.

\section{E - Via de administraçắo da antitoxina:}

Nos vários estudos é utilizada a via lom. bar ou a via cisternal. Embora, em Mesa Ro. donda durante a Quarta Conferência Interna cional sobre Tétano ${ }^{8}$, seja preferida a via cisternal, nấo há confirmação da sua superioridade, como demonstra o estudo de SANDERS et al. 32 .

\section{F - Complicaçōes do uso da antitoxina por via intratecal:}

A partir de 1941 houve um posicionamen. to de vários autores ${ }^{41}$ contra o uso de SAT por via intratecal, em virtude da alta incidên. cia de complicações graves atribuidas a esta via: choque, desencadeamento de espasmos e apnéia.

Atualmente, com a maior purificação da antitoxina heteróloga e com o uso da antito xina homóloga, os efeitos colaterais são menos freqüentes e menos intensos. São descritas complicações despreziveis ${ }^{3}$ ou leves, ca mo náuseas, vômitos, cefaléia e síndrome de irritação meníngea com TIG 5,46 .

Com SAT são descritas: náuseas, vômitos, cefaléia, suores, e alterações psicomotoras lo ves a moderadas 6,21,48.

ROBERT et al. ${ }^{30}$ descrevem paraplegia em membros inferiores que se inicia algumas ho. 
LEVIN, A. S. S.; BARONE, A. A. \& SHIROMA, M. - Soroterapia intratecal no tétano. Revisäo. Rev. Inst. Med. trop. são Paulo, 29:255-267, 1987.

T A B E I A IV (4." parte)

Resultados de estudos realizados com soro antitetânico (ie origem equina (SAT) por via intratecal associado à via sistêmica, compilados da literatura

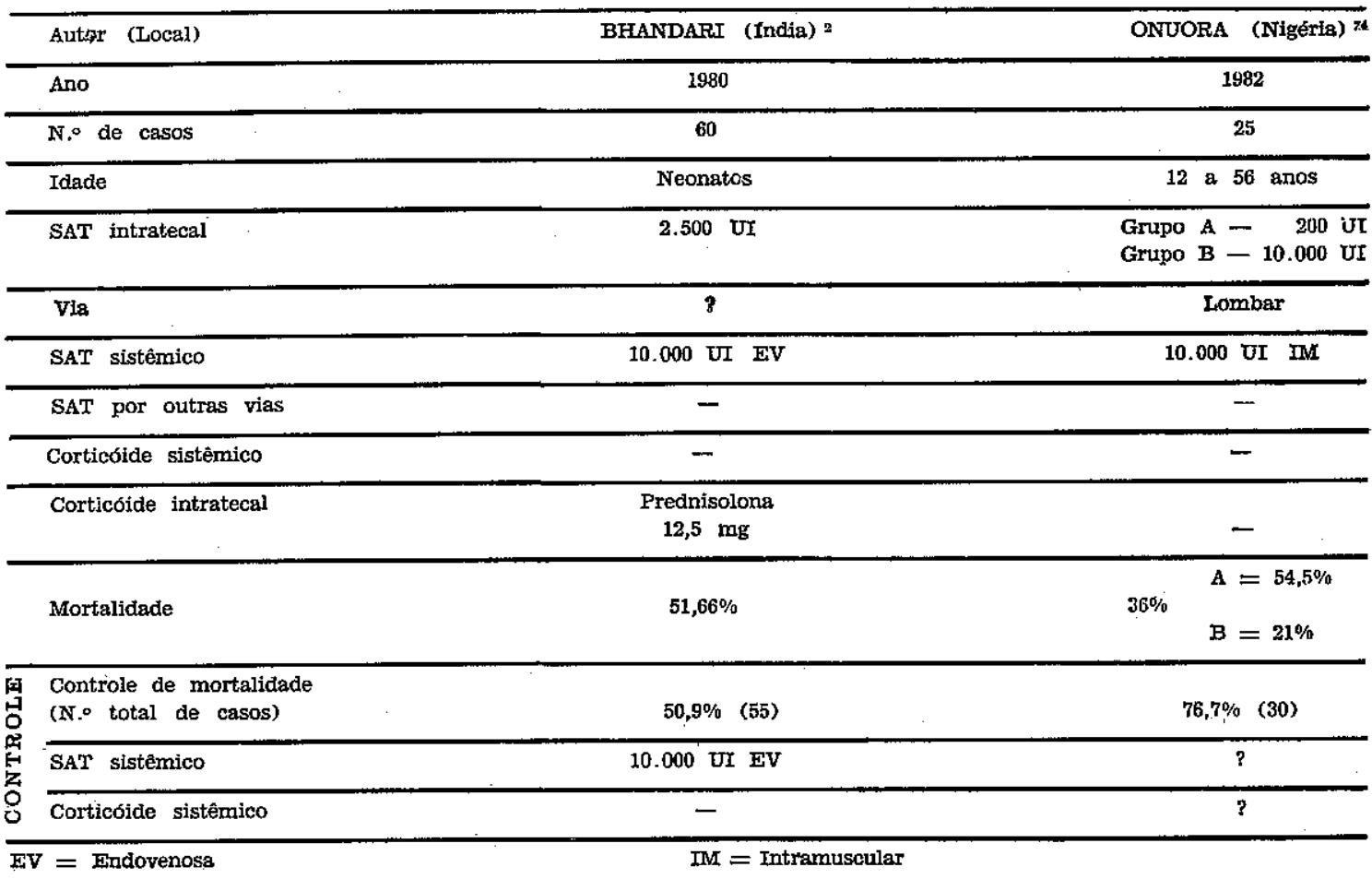

ras após a injeção intratecal de antitoxina homóloga, cujo preservativo é tiomersal, e que é reversível até 12 horas após o seu início. Também encontram alteração liquórica, com pequeno e transitório aumento de celularidade e aumento de proteinorraquia, que persiste até urn mês após a injeção. Levantam eles, a possibilidade de também haver anafilaxia, o que explicaria reações adversas imediatas à injeção.

ILDIRIM ${ }^{20}$, em experiências com cães, de monstra que as complicaçōes da soroterapia intratecal são atribuíveis ao fenol, usado co. mo preservativo. Esse autor acredita que o uso de tiomersal na concentração $1: 10.000$ eै inócuo, porém nāo faz estuđo liquórico.

Para muitos autores, o uso de corticóide por via intratecal associado à antitoxina ${ }^{18}$, assim como por via sistêmica, tem a função de diminuir os efeitos colaterais.

\section{G $-O$ uso da via peridural da antitoxina:}

TESTASOCCA et 21.42 , em 1984, são favoráveis à associação đa via peridural à via sistêmica da antitoxina. Utìlizando essa via em 28 pacientes, obtém mortalidade de $43 \%$, mas são necessários estudos complementares para obter conclusões mais concretas.

\section{COMENTARIOS FINAIS}

Tétano é uma doença de grande importância, principalmente em paises em desenvolvimento. Nas regiōes em que a sua incidência é elevada, geralmente não existem recursos terapêuticos avançados, como Terapia Intensiva e enfermagem especializada. Nessas condiçōes a mortalidade por essa doença é alta, utilizan. do-se a terapêutica clássica, ou seja, a antitoxina por via sistêmica.

Utilizar a via intratecal, para administrar ção da antitoxina, seria uma maneira de encurtar o tempo de internação e diminuir a gravidade do tétano. Dessa maneira prescindir de cuidados hospitalares mais intensos, ine xistentes em muitos locais, e, com isso tentar reduzir a mortalidade pela doença.

A antitoxina tetânica por via intrarraquu. diana agiria neutralizando a toxina já fixada 
LEVIN, A. S. S.; BARONE, A. A. \& SHIROMA, M. - Soroterapia intratecal no tétano. Revisão. Rev. Xnst. Med. trop. São Paulo, 29:255-267, 1987.

T A B E L A IV (5." parte)

Resultados de estudos realizados com soro antitetânico de origem equina (SAT) por via intratecal associado à via sistėmica, compilados da literatura

\begin{tabular}{|c|c|c|c|}
\hline & Autar (Local) & THOMAS (fndia) ${ }^{43}$ & THOMAS (India) \\
\hline & Ano & 1982 & 1982 \\
\hline & N..$^{\circ}$ de casos & 81 & 45 \\
\hline & Idade & A. partir de 12 anos & A partir de 12 anos \\
\hline & SAT intratecal & $200 \mathrm{VI}$ & 200 UI \\
\hline & Via & $?$ & $?$ \\
\hline & SAT sistêmico & 2.250 UI IM & $2.250 \mathrm{UI} \mathrm{IM}$ \\
\hline & SAT por outras vias & $\rightarrow$ & - \\
\hline & Corticóide sistêmico & - & $\begin{array}{l}\text { Betametasona } \\
16 \text { a } 24 \mathrm{mg} \text { EV/dia }\end{array}$ \\
\hline & Corticóide intratecal & $\begin{array}{l}\text { Fidrocortisona } \\
100 \mathrm{mg}\end{array}$ & - \\
\hline & Mortalidade & $53 \%$ & $27 \%$ \\
\hline 国 & $\begin{array}{l}\text { Controle de mortalidade } \\
\text { (N.० total de casos) }\end{array}$ & $63 \% \quad(73)$ & $63 \% \quad(73)$ \\
\hline 夏 & SAT sistêtmico & 10.000 UI IM & 10.000 UI IM \\
\hline 0 & Corticóide sistêmico & - & - \\
\hline
\end{tabular}

TA B E L A IV (6.a parte)

Resultados de estudos realizados com soro antitetânico de ortgem equina (SAT) por via intratecal associado à via sistêmica, compilados da Ifteratura

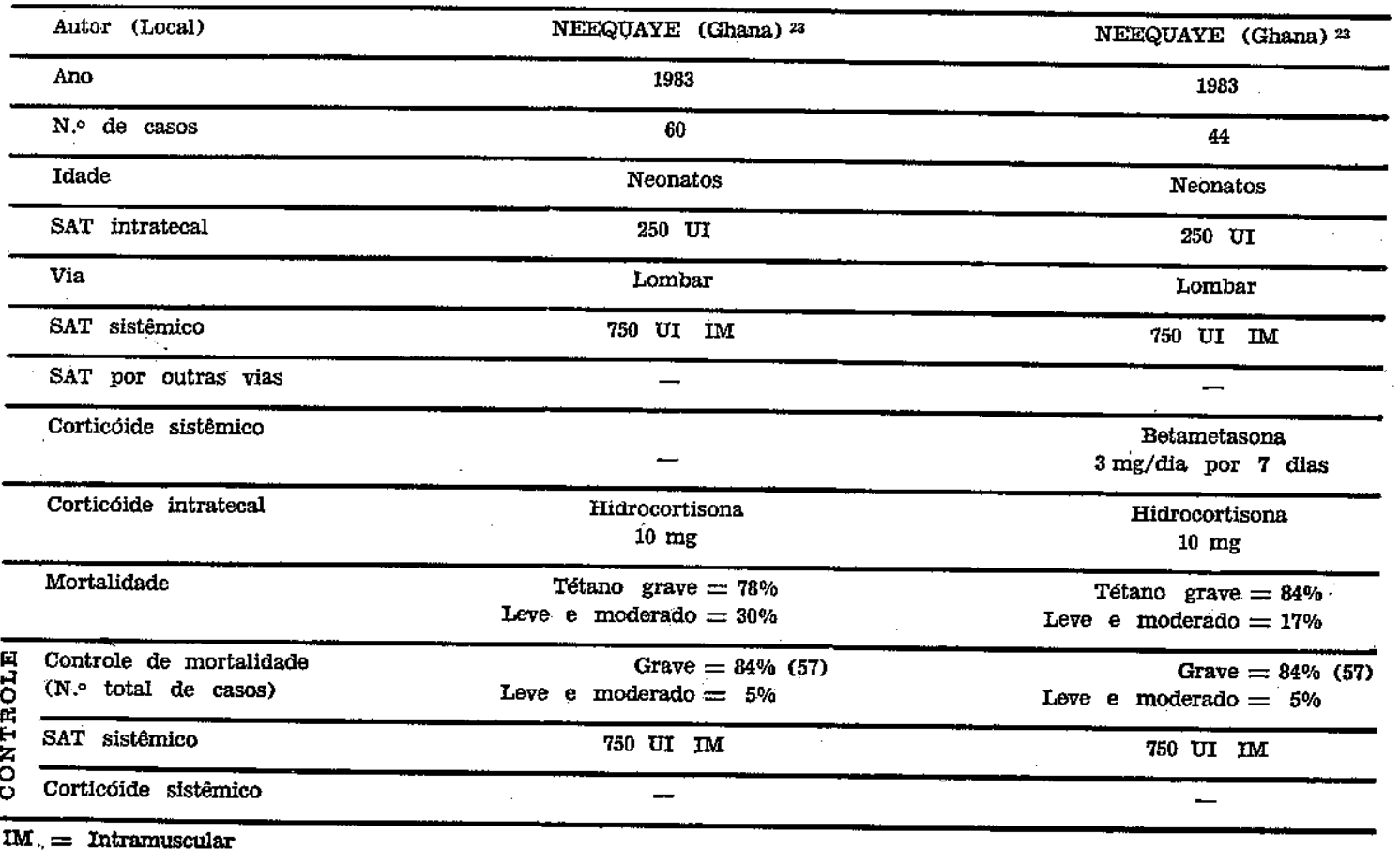


LEVIN, A. S. S.; BARONE, A. A. \& SHIROMA, M. - Soroterapia intratecal no tétano. Revisāo. Rev. Inst. Med. trop: São Paulo, 29:255-267, 1987.

no SNC e a toxina livre no líquido céfalo-raquidiano, em virtude de seu acesso direto sem ter que ultrapassar a barreira hemato-liquorica. Esse mecanismo ainda é teórico.

Há um grande número de estudos clínicos nessa área. Embora muitos deles não sejam bem controlados e não possam ser considerados conclusivos, outros obtém resultados conflitantes. Estudos feitos com antitoxina homóloga (TIG) por GUPTA ${ }^{14}$, em tétano leve ou inicial, DIOP-MAR et al. 5 , no Senegal, e LIST 22, na Austria, obtém diminuição significativa de mortalidade pela doença utilizando a sua administração intratecal. No entanto, estudos, também com antitoxina homóloga, por BOLOT et al.3.4, na França, SEDAGHATIAN 35 , e VALKI et al. $44,45,46$, não demonstram vantagem do seu uso por via intratecal. $O$ mesmo conflito existe entre estudos que utilizam antitoxina de origem equina (SAT). GALLAIS et al. 10,11,12, na Costa do Marfim, SINGH et al. ${ }^{38}$, KESWANI et al. ${ }^{21}$, os dois últimos trabalhos em tétano leve ou inicial, e SANDERS et al. 32 , consideram os resultados, obtidos com o uso da antitoxina pela via intratecal, significativamente melhores do que aqueles obtidos nos seus grupos controle. NEEQUAYE et al. ${ }^{23}$, THOMAS et al. ${ }^{43}$, e BHANDARI et al. ${ }^{2}$, por sua vez, não demonstram vantagem na administração de SAT por via intratecal. Múltiplas variáveis existem que poderiam explicar essas diferenças de resultar dos: o uso sistêmico ou intratecal de corticóides, o uso de antitoxina homóloga ou heteróloga a sua posologia, a sua via de administração.

As complicaçōes que advem do uso da antitoxina por via intrarraquidiana são, geralmente, leves e transitórias, e não contraindicam o seu uso.

Em alguns trabalhos 28,29,36, há tentativas de incluir definitivamente a administração intratecal de antitoxina, no tratamento do tétano. Essas conclusões nos parecem precoces. O assunto exige, ainda, estudos prospectivos bem controlados, e que levem em consideração as muitas variáveis envolvidas, para trazer conclusões concretas.

\section{SUMMARY}

Intrathecal antitoxin in the treatment of tetanus.
Tetanus is an important disease in underdeveloped countries. We present a review of literature concerning the use of intrathecal antitoxin in the treatment of tetanus. Historical aspects and the physiopathologic basis for this route of administration of antitoxin are brought up and the efficacy of the uses of heterologous and homologous antitoxin associated or not to systemic antitoxin and steroids is discussed.

A great number of clinical studies have been done and many aren't well controlled or conclusive. Others have conflicting results. Homologous antitoxin (TIG) was used by GUPTA ${ }^{14}$, in slight cases of tetanus; DIOP-MAR et al ${ }^{5}$, in Senegal; and LIST 2 , in Austria, with a reduction of the mortality by the disease when of its intrathecal use. However, BOLOT et al 3,4, in France; SEDAGFATIAN ${ }^{35}$, and VALKI et al 44,45,46, don't obtain favorable results with intrathecal TIG. The same controversy arises with the use of intrathecal heterologous antitoxin (SAT). GALlAIS et al 10,11,12; SINGH et al ${ }^{38}$; KESWANI et al ${ }^{21}$; SANDERS et al ${ }^{32}$, obtain significantly better results when compared to their control groups. NEEQUAYE et al ${ }^{23}$; THOMAS et al ${ }^{43}$; and BHANDARI et $\mathrm{al}^{2}$, find no advantages to the use of this route.

There are a number of factors possibly responsible for these differences in results: the intrathecal or systemic use of steroids; the use of homologous or heterologous antitoxin; their dosage and route of administration.

The adverse effects due to intrathecal antitoxin are in general slight, however this route of administration should be considered experimental until there is further evidence of its benefit.

\section{REFERENCIAS BIBLIOGRAFICA:}

1. ARNAUD, P. H.; CHARMEAU, A.; BOLOT, J. F. \& FOURNIER, G. - Exploration de la sérothérapie intrathécale dans le tétanos par la méthode des traceurs nucléaires. In: INTERNATIONAL CONFERENCE ON TETANUS, 4., Dakar, 1975. Proceedings. Lyon, Fondation Merleux, 1975. p. $427-453$.

2. BHANDARI, B.; AJMERA, N. K. \& JAGETIYA, P. R. - Intratecal anti-tetants serum in management of tetanus neonatorum. Indian J. med. Res., 72: 685-687, 1980.

3. BOLOT, J. F.; CANTON, P. H.; CARDINAUD, J. P.; STELLMANN, C. \& TRIAU, R. - Serothérapie intrathécale par gammaglobulines antitétaniques humaines. 
LEVIN, A. S. S.; BARONE, A. A. \& SHIROMA, M. - Soroterapia intratecal no tétano. Revisão. Rev. Inst. Med. trop. Săo Paulo, 29:255-267, 1987 .

étude randomisée de 87 cas. In INTERNATIONAr CON FIRENCE ON TETANUS, 4., Dakar, 1975. Proceedings. Lyon, Fondation Merieux, 1975. p. $455-460$.

4. BOLOT, J. F.; FOURNIER, G.; CANTON, R.; CARDINAUD, J. P.; REY, M.; STELTMAN, C.; TRIAU, $R$. \& DIOP.MAR, I. - Intrathecal (I.T) human specific antitetanus (H.S.A.G.). A multicentric controlled trial (Abstract). In: INTERNATIONAL CONFERENCE ON TETANUS, 5., Ronneby, Sweden, 1978. Sumaries of the presentations. Ronneby, 1978. p. 75.

5. DIOP-MAR, I.; BADIANE, S.; DIAGNE, S.; STELLMANN, C. \& REY, M. - Confírmation de l'efficacité de la sérothérapie intrathécale par une étude randomisé de 245 cas. In: INTERNATIONAL CONFERENCE ON TETANUS, 4., Dakar, 1975. Proceedings. Lyon, Fondation Merieux, 1975. p. $461-465$.

6. DIOP-MAR, I.; BADIANE, S. \& BA, A. - Traitment du tétanos par le sérum antitétanfque hetérologue intrathécal. Bull. Soc. méd. Afr. noire Langue franç, 23: $393-401,1978$.

7. DIOR-MAR, I.; BADIANE, S.; SOW, A. \& BA, A. Treatment of tetanus by intrathecal heterologous antitoxim (Abstract). In: INTERNATIONAL CONFIRRENCF ON TETANuS, 5., Ronneby, Sweden, 1978, Summarles of the presentations. Ronneby, 1978. p. 71.

8. DƯREUX, J. B., modérateur - Table ronde: Progrès dans la sérothérapie du tétanos déclaré. In INTERNATIONAL CONFERENCE ON TETANUS, 4., Dakar, 1975. Proceedings. Iyyon, Fondation Merieux, 1975. p. 467-484.

9. FLOREY, H. \& FilDES, P: - Tetanus VIII. The treatment of tetanus in rabbits by large intrathecal doses of antitoxin. Brit. J. exp. Path., 8: 393-397, 1927.

10. GaLLAIS, H. - Intérät de I'administration intrathécale de sérum antitétanique et de corticoides pour le tralment du tétanos déclaré. Nouv Presșe méd, 6: 1571, 1977.

11. GALlaIS, H.; MOREAU, J.; CORNET, C.; ODEHOURT, K.; ABISSE, A. \& ECHIMANE, A. - Therapeutic evaluation of 404 cases of tetanus - Advantages of intrathecal serotherapy (Abstract). In: INTERNATIONAL CONFERENCE ON TETANUS, 5., Ronneby, Sweden, 1978. Summaries of the presentations. Ronneby', 1978. p. 73 .

12. GaILAIS, H.; ADAMA, S. \& CASANOVA, P. - Our practical experience of intrathecal serotherapy obtained from a series of 727 cases of tetanus (Abstract). In; NISTICO, G. \& STRONGOLI, M. C., ed. - International Conference on Tetanus, 7., Copanello, Italy, 1984. Abstracts. Copanello, 1984. p. 130.

13. GRECCO, J. B. - Antitetanus serum and prednisolone in the intrathecal treatment of puerperal and umbilical tetanus. In: INTHERATIONAT CONFBRENCE ON TE. TANUS, 4., Dakar, 1975. Proceedings. Lyon, Fondation. Merleux, 1975. p. 415-421.

14. GUPTA, P. S.; GOYAL, S.; KAPOR, R.; BATRA, V. $\mathbf{K}$. \& JAIN, B. K. - Intrathecal human tetanus immuno. . globulin in early tetanus. Lancet, 2: 439-440, 1980.
15. HANAUSHE, A.; ERDMANN, G. \& WhLLFÖRR, H. H. - $F\left(a b^{\prime}\right)_{2}-$ Tetanus - antiserum: distribution after intrathecal injection and further purification by affinity chromatography. In: INTERNATIONAL CONFERENCE ON TETANUS, 5., Ronneby, Sweden, 1978. Summaries of the presentations. Ronneby, 1978. p. 52.

16. HENDERSON, A.; GUY, P. \& KNIGHT, J. R. - Severe tetanus complicating an open fernoral fracture successfully managed using intrathecal human tetanus immunoglobulin and external fixation. J. roy Army med. Cps., 130: 185-187, 1984.

17. HUGUES-DAVIES, T. H. - Intrathecal immunoglobulin for tetanus (letter). Med. J. Aust., 2: 308, 1979.

18. IDDIRIM, I.; MEIRA, A. R. \& FURCOLOW, M. L. Tetanus (letter). New Engl. J. Med., 280: 1243, 1969.

19. IUDIRIM, I. - Intrathecal treatment of tetanus with antitetanus serum and predinisolone mixture. (In: EDSALL, G., ed. - INTERNATIONAL CONFERENCE ON TETANUS, 3., Sāo Paulo, 1970. Procedings. Washington, PAHO; WHO, 1972. p. 119-127.

20. IUDIRIM, I. - General and intrathecal serotherapy. In: INTERNATIONAL CONEERENCE ON TETANUS, 4, Dakar, 1975. Proceedings: Lyon, Fendation, Merieux, 1975. p. 371-382.

21. KESWANI, N. $\mathbf{K}$; SINGH, A. K. \& UPADHYAYA; $\mathbf{K}$. D. - Intrathecal tetanus antitoxin in moderate and severe tetanus. J. Indian med. Ass., "55: 67-69, 1980.

22. LIST, W. F. - Tetanus treatment with high doses of tetanus antitoxin (Abstract). In: INTFRNATrONAI. CONEFRENCE ON TETANUS, 5., Ronneby, Sweden, 1978. Summaries of the presentations. Ronneby, 1978. p. 72 .

23. NEEQUAYF, J. \& NKRUMAF, F. K. - Failure of intrathecal antitetanus serum to improve survival in neonatal tetanus. Arch. Dis. Childh,, 58: 276-278, 1983.

24. ONUORA, C. A.; SAMBO-DONGA, L. \& YAFAYA, H. - Intrathecal antitetanus serum in the management of tetanus. E. Afr. med. J., 59: 52-55, 1982.

25. PARK, W.; NICOLL, M. - Experiments on the curative value of the intraspinal administration of tetanus an. titoxin. J. Amer. med. Ass., 65: 235-238, 1914.

26. PATEL, J. C.; MFHTA, B. C.; GOODLUCK, P. L.; RAO, S. S. \& BORKAR, M. S. - Detection of free circulating toxin the blood and cerebrospinal fluid of some tetanus cases and its significance (Abstract). In: NISITCO, G. \& STRONGOLI, M. C., ed. - INTERNATIONAL, CONFERENCE ON TETANUS, 7., Copanello, Italy, 1984. Abstracts. Copanello, 1984, p. 114.

27. PENNA - Subaracnoid serum treatment of tetanus. J. Amer. med. Ass., 38: 602, 1902.

28. REINHARDT, F. - Intravenous and intrathecal application of tetanus antitoxim of human origin. In: INTERNATIONAL CONFHRENCE ON TETANUS, 5., Dakar, 1975. Proceedings. Lyon, Fondation Merieux, 1975. p. $409-413$. 
LEVIN, A. S. S.; BARONE, A. A. \& SHIROMA, M. - Soroterapia intratecal no tétano. Revisão. Rev. Inst. Med. trop. São Paulo, 29:255-267, 1987.

29. REY, M.; DIOP-MAR, I. \& ROBERT, D. - Treatment of Tetanus. In: VERONESI, R. - Tetanus: important new concepts. Amoterdam, Faxcerpta Medica, 1981. p. 207-237.

30.. ROBERT, R.; ROUFFINEAU, J.; CREMAULT, A.; BAUPLE, J. L.; POURRAT, O.; GIL, R. \& PATTE; D. - Paraplégie réversible après injection intrathécale de fortes doses de gammaglobulines humaines lors du trait. ment de tétanos de faible gravité - quatre observations. Presse méd., 13: 1947-1949, 1984.

31. ROGHRS, J. - The treatment of tetanus by intraneural and intraspinal injections of antitoxin. J. Amer. med. Ass., 45: 12-18, 1905.

32. SANDERS, R. K. M.; JOSEPE, R.; MARTYN, B. \& PEACOCK, M. L. - Intrathecal antitetanus serum (horse) in the treatment of tetanus. Lancet, 1: 974-977, 1977.

33. SANDERS, R. K. M. - Treatment of tetanus (letter). Brit. med. J., 2: 1979

34. SANDERS, R. K. M. - Intrathecal tetanus serum (letter). Lancet, 2: 1200, 1980.

35. SEDAGHATIAN, M. R. - Intrathecal serotherapy in neonatal tetanus: a controlled trial. Arch. Dis. Childh., 54: $623-625,1979$.

36. SHANN, F. - Intrathecal administration of tetanus antiserum (letter). Med. J. Aust., 24: 604-605, 1983.

37. SHERRINGTON, C. S. - Observations with antitetanus serum in the monkey. Xancet, 2: 964-966, 1917.

38. SINGF, A. K.; BANSAL, A.; GOEL, S. P. \& AGARWAL, V. K. - Intrathecal antitetanus serum (horse) with steroid in the treatment of neonatal tetanus. Arch. Dis. Child., 55: 527-531, 1980.

39. SINGHr, S. \& SINGHI, P. - Intrathecal ATS and high dosage diazepan in neonatal tetanus (letter). Arch. Dis. Child., 54: 650-651, 1979.

40. SMITH, J. W. G. - Intracerebral antitoxin experimental tetanus. Brit. J. exp. Path, 47: 17-24, 1966.

41. SPAETH, R. - Therapy of tetanus - a estudy of two-hundred and seventy-six cases. Arch. intern. Med., 68: 1133-1160, 1941.

42. TESTASOCCA, D.; TORRONI, P.; MAZZONI, A. L.; ZENOBI, G. \& GUAZZINI, S. - Advancement in treating of tetanus: intravenous and peridural administration of human immunoglobulins (Abstract). In: NISTICO, G. \& STRONGOLI, M. C., ed. - International Conference on Tetanus, 7., Copanello, Italy, 1984 Abstracts. Copanello, 1984. p. 133.

43. THOMAS, P. P.; CROWELL, E. B. \& MATHEW, M - Intrathecal anti-tetanus serum (ATS) and parenteral betamethasone in the treatment of tetanus. Trans. roy. Soc. trop. Med. Hyg., 76: 620-623, 1982.

44. VAKIL, B. J.; ARMITAGE, P. \& LAURENCE, D. R - Therapeutic trial of intrathecal human tetanus immunoglobulin in severe tetanus. A preliminary com munication. In: INTERNATIONAL CONFERENCE ON TETANUS, 4., Dakar, 1975. Proceedings. Lyon, Fondation Merieux, 1975. p. $423-425$.

45. VAKIL, B. J.; ARMITAGE, P.; CLIFFORD, R. E. LAURENCE, D. R. - Therapeutic trials of intrathecal (intracisternal) administration of human tetanus immunoglobulin in severe tetanus. (Aibstract). In INTERNATIONAI CONFERENCE ON TETANUS, 5., Ronneby, Sweden, 1978. Summaries of the presentations. Ronneby, 1978 , p. 74 .

46. VAKIL, B. J.; ARMITAGE; P.; CLIFFORD, R. E. \& LAURENCE, D. R. - Therapeutic trial of intracisternal human immunoglobulin in clinical tetanus. Trans. roy. Soc. trop. Med. Hyg., 73: 579-583, 1979.

47. VERONESI, R.; BIZZINI, B.; HUTZLER, T. U.; FO CACCIA, R.; MAZZA, C. C.; FELDMAN, C.; FERREIRA, M. S.; DIETZE, R.; RODRIGUES, M. L. M. \& Tr MERMAN, A. - Eficácia do tratamento do tétano com antitoxina tetanica por via raquidiana e/ou vonosa. Estudo de 101 casos, com pesquisa sobre a permanência da gamaglobulina humana $F(a b)^{2}$ no liquor e no sangue. Rev. bras. Clin. Terap., 9: 301-319, 1980.

48. VERONESI, R.; BIZZINI, B.; MAZZA, C. C.; FOCACCIA, R.; FELDMAN, C.; COSCINA, A. L. \& HUTZLER, R. U. - Tratamento especifico do tétano com uma fraçāo $F\left(a b^{\prime}\right)$ da imunoglobulina antitetânica injetada por via raquidiana. Kev. Hosp. Clín. Fac. Med. S. Paaulo, 38: 147-149, 1983 .

49. WHLLHONER, H. H.; NIGGEMANN, B. \& ERDMANN, G. - Spinal pharmacokinetics of liposome-entrapped tetanus antibodies. (Abstract). In: : NISTICO, G. \& STRANGOLI, M. C., ed. - INTERNATIONAI CON. FERENCE ON THEANUS, 7., Copanello, Italy, 1984. Abstracts. Copanello, 1984. p. 131.

Recebido para publịcsçăo 24/3/87. 